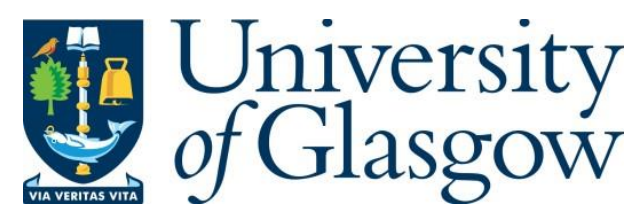

Schmidt, C. et al. (2018) How reproducible are kinetic parameter constraints of quartz luminescence? An interlaboratory comparison for the $110^{\circ} \mathrm{C}$ TL peak. Radiation Measurements, 110, pp. 14-24. (doi:10.1016/j.radmeas.2018.01.002)

There may be differences between this version and the published version. You are advised to consult the publisher's version if you wish to cite from it.

http://eprints.gla.ac.uk/167142/

Deposited on: 20 August 2018

Enlighten - Research publications by members of the University of Glasgow http://eprints.gla.ac.uk 


\title{
How reproducible are kinetic parameter constraints of quartz luminescence? An interlaboratory comparison for the $110{ }^{\circ} \mathrm{C}$ TL peak
}

\author{
Christoph Schmidt ${ }^{1}$, Johannes Friedrich ${ }^{1}$, Grzegorz Adamiec ${ }^{2}$, Alicja Chruścińska ${ }^{3}$, Mauro
}

5 Fasoli $^{4,5}$, Sebastian Kreutzer ${ }^{6}$, Marco Martini ${ }^{4,5}$, Laura Panzeri ${ }^{4,5}$, Georgios S. Polymeris ${ }^{7}$, Krzysztof Przegietka ${ }^{3}$, Pierre G. Valla ${ }^{8}$, Georgina E. King ${ }^{8}$, David. C.W. Sanderson ${ }^{9}$

${ }^{1}$ Chair of Geomorphology \& BayCEER, University of Bayreuth, 95440 Bayreuth, Germany

${ }^{2}$ GADAM Centre, Institute of Physics, Silesian University of Technology, ul. Krzywoustego 2, 44-100 Gliwice, Poland

${ }^{3}$ Institute of Physics, Faculty of Physics, Astronomy and Informatics, Nicolaus Copernicus University, Grudziadzka 5/7, 87100 Torun, Poland

${ }^{4}$ Dipartimento di Scienza dei Materiali, Università degli Studi di Milano-Bicocca, via Cozzi 55, IT-20125 Milan, Italy

${ }^{5}$ INFN, Sezione di Milano-Bicocca, Piazza della Scienza 3, IT-20126 Milan, Italy

${ }^{6}$ IRAMAT-CRP2A, Université Bordeaux Montaigne, Pessec Cedex, France

${ }^{7}$ Institute of Nuclear Sciences, Ankara University, 06100 Beşevler-Ankara, Turkey

${ }^{8}$ Institute of Geological Sciences and Oeschger Centre for Climate Change Research, University of Bern, Baltzerstr. 1+3, 3012 Bern, Switzerland

${ }^{9}$ Scottish Universities Environmental Research Centre, Rankine Avenue, East Kilbride G75 0QF, United Kingdom

20 Abstract

Knowledge of the kinetic parameters $E$ (thermal activation energy) and $s$ (frequency factor) of charge-trapping defects in the quartz crystal lattice is of paramount importance to assess the thermal stability of associated luminescence signals used for dosimetry and dating. Since methods proposed for constraining thermoluminescence (TL) kinetics usually make use of the signal response to thermal treatments, accurate temperature control is required to obtain valid $E$ and $s$ values. In an attempt to check the extent to which consistent kinetic parameters could be obtained using routine luminescence measurement equipment, we have investigated three methods (isothermal decay, initial rise and the Hoogenstraaten method) in an intercomparison study involving eight laboratories using Risø and Freiberg Instruments systems. The target signal was the so-called $110^{\circ} \mathrm{C}$ TL peak of a sample of Oligocene coastal dune quartz sand from the Fontainebleau sand formation (France).

TL glow curves recorded with heating rates in the range $0.02-5.0 \mathrm{~K} \mathrm{~s}^{-1}$ showed peak positions varying by up to $60{ }^{\circ} \mathrm{C}$ between systems at the highest heating rates, as caused by temperature calibration errors and/or thermal lag. Kinetic parameters derived from the complete data set show a large spread, covering the ranges $\sim 0.5-1.2 \mathrm{eV}$ and $10^{5}-10^{18} \mathrm{~s}^{-1}$ for $E$ and $s$. In most cases, interlaboratory variations exceeded those of replicate measurements within individual laboratories. Signal lifetimes at $20^{\circ} \mathrm{C}$ derived from the isothermal decay ( $\left.\sim 57 \mathrm{~min}\right)$ and initial rise methods (at low heating rates; $\sim 60-80 \mathrm{~min}$ ) most closely match the directly measured value ( $\sim 70 \mathrm{~min})$. Finally, we discuss the consequences of these findings for dosimetry and 
dating using luminescence signals and possible ways to reduce systematic errors in laboratory measurements of kinetic parameters.

Keywords: Thermoluminescence; Kinetic parameters; Trap depth; Frequency factor;

\section{Introduction}

In recent decades, thermoluminescence (TL) and optically stimulated luminescence (OSL) dating of natural minerals such as quartz and feldspar have become two of the most important Quaternary chronometric methods. Like for all other methods of trapped charge dating, a prerequisite for accurate and reliable dating results is a sufficiently high thermal stability of the dating signal, i.e., of the signal generated by recombination of electrons thermally or optically released from their traps (see, e.g., Aitken, 1985; Preusser et al., 2009 or Chen and Pagonis, 2011 for physical basics of the luminescence process). For quartz, the luminescence signal stability is thought to be purely a first-order function of the ambient temperature and the physical parameters describing the thermal stability of trapped charge for a particular type of trap. In the case of feldspars, however, the signal can decrease over time also due to socalled anomalous fading (likely caused by quantum-mechanical tunnelling processes; e.g., Wintle, 1973; Visocekas, 1985; Huntley and Lamothe, 2001; Huntley, 2006) in addition to thermal depletion, which itself is not a first-order process. In the absence of anomalous fading, the electron lifetime (or electron retention time) $\tau$ in a trap is commonly expressed using the following formula (Aitken, 1985):

$$
\tau=S^{-1} \cdot e^{\frac{E}{k_{B} T}}
$$

where $s$ is the frequency factor (in $\mathrm{s}^{-1}$; describing the interaction of electron and crystal lattice), $E$ is the thermal activation energy needed to release the electron from the trap (in $\mathrm{eV}$; may differ from the one for other excitation modes, as discussed in Chen and McKeever, 1997), $k_{\mathrm{B}}$ is the Boltzmann constant (in $\mathrm{eV} \mathrm{K}^{-1}$ ) and $T$ is the absolute temperature (in K). Aitken (1985) proposed that for application in dating, the electron retention time (i.e., the lifetime) at storage temperature should be at least ten times as long as the dating period to reduce age underestimation to $<5 \%$. 
75 It thus becomes clear that accurate knowledge of the trap parameters $E$ and $s$ is fundamental to calculate the thermal stability of a specific luminescence signal (sourced from a particular type of trap) and to avoid any systematic errors in age determination. Alongside chronometric applications, luminescence of quartz and feldspar has recently been proposed and successfully employed for thermochronometry, i.e., for reconstructing the thermal history of rocks

80 (Herman et al., 2010; Guralnik et al., 2015; Schmidt et al., 2015; King et al., 2016a,b,c). Since the sensitivity of the relative trap filling level (i.e., the rates of electron capture in relation to electron release) to changes in ambient temperature is the basic principle of this method, the accuracy of the input parameters $E$ and $s$ for the targeted electron trap critically determines the inversion of relative trap filling level into a thermal history.

A range of various methods was proposed for determining the kinetic parameters of TL and OSL signals; summaries are given for instance in Mahesh et al. (1989), Chen and McKeever (1997) and Chen and Pagonis (2011). All of these methods make use of the luminescence signal response to thermal treatments (e.g., the Hoogenstraaten method, the initial rise method or isothermal decay; see Section 3) and, hence, accurate temperature control in the experiments is required to obtain reliable results. However, it is known that systematic offsets between target temperature and actual sample temperature occur due to both thermal lag and inaccurate temperature calibration of the heating element. The effect of thermal lag results from the thermal inertia of the heating unit, the sample carrier, the sample itself and the adhesive often used to fix the mineral grains. Poor thermal contact between heater and sample carrier can significantly increase thermal lag as is also the case for high heating rates (e.g., Kitis et al., 1999, 2015). Minimising or correcting for thermal lag should therefore be taken into account when kinetic parameters are to be measured.

For many different TL and OSL signals of quartz, trapping parameters $E$ and $s$ were published (TL: e.g., Wintle, 1975; Fleming, 1979; OSL: e.g., Huntley et al., 1996; Singarayer and Bailey, 2003). In view of the exponential dependence of the lifetime $\tau$ on thermal activation energy $E$, the following questions arise:

1. Can literature values for $E$ and $s$ be adopted as standards for all calculations in the context of dosimetry, dating and thermochrnometry, given the technological development in luminescence dating equipment?

2. How large is the influence of laboratory-specific measurement conditions (e.g., type of sample carriers, temperature calibration) on resulting trapping parameters? 
To address these questions and in an attempt to check to which extent consistent kinetic

110 parameters can be obtained using routine luminescence dating equipment, we investigated three methods for quantifying $E$ and $s$ from a reference quartz sample in an interlaboratory comparison involving eight laboratories. The so-called $110^{\circ} \mathrm{C}$ TL peak (recorded in the UV spectral range; 280-380 nm) was chosen as target signal, because it is omnipresent in quartz samples and shows widely accepted first-order kinetic behaviour (Pagonis et al., 2003; see also HTML file 1 in the supplementary material, where experimental data for the $110^{\circ} \mathrm{C} \mathrm{TL}$ peak were fitted with a first-order kinetic function). Its lifetime at room temperature is in the order of tens of minutes and thus excludes its use for (direct) dating purposes. However, a strong pre-dose effect was observed (Zimmerman, 1971) and exploited extensively for pottery dating, authentification testing and accident dosimetry (e.g., Fleming, 1973; Bailiff and Huskell, 1983; Bailiff, 1994). It was further suggested to assess the heat treatment of archaeological materials by use of the temperature-dependent sensitisation of this emission (Sunta and David, 1982; Watson and Aitken, 1985; Göksu et al., 1989; Koul et al., 1996; Godfrey-Smith and Ilani, 2004). In terms of OSL dating, the sensitisation of the $110^{\circ} \mathrm{C}$ TL peak has been shown to correlate with that of the initial portion of the OSL decay curve, allowing the use of the $110^{\circ} \mathrm{C}$ TL signal for sensitivity correction in regenerative OSL protocols (Stoneham and Stokes, 1992; Chen et al., 2000; Jain et al., 2003).

The outcomes of this study provide important information on the reproducibility of absolute physical parameters obtained from luminescence measurements of identical batches of a reference quartz sample among different laboratories. In that sense, the present investigation

130 differs from previous interlaboratory dating comparisons, which are based on relative/normalised luminescence measurements (e.g., Murray et al., 2015).

\section{Literature values of trapping parameters for the quartz $110{ }^{\circ} \mathrm{C}$ TL peak}

135 Previously published $E$ and $s$ values for the $110^{\circ} \mathrm{C}$ TL peak in quartz were obtained using different methods, from which those relevant for this investigation are briefly described in Section 3. Table 1 contains a (non-exhaustive) summary of literature values, including the type of quartz investigated and further measurement parameters (e.g., detection filter, heating rate, equipment) considered as relevant with regard to the present study. Wherever possible,

140 the corresponding lifetime $\tau$ at $20^{\circ} \mathrm{C}$ was calculated for each set of $E$ and $s$.

\section{Table 1}


Values for $E$ and $s$ span the range between 0.60 and $1.05 \mathrm{eV}$ and between $2.7 \times 10^{9}$ and $4.7 \mathrm{x}$ $10^{13} \mathrm{~s}^{-1}$, respectively. Calculated lifetimes vary between $16 \mathrm{~min}$ and $\sim 6 \mathrm{~h}$, one outlier yields

$\sim 70 \mathrm{~h}$. Since the experimental parameters used in the cited studies - such as the quartz sample itself, the equipment or the laboratory procedures - were very different, the variability of trapping parameters as shown in Table 1 is difficult to interpret.

\section{Materials and methods}

\subsection{Sample and sample preparation}

The reference quartz sample used for the comparative kinetic study was extracted from an Oligocene coastal dune sand from the Fontainebleau sand formation (France; lab code FB).

155 The sample was sieved to grain sizes in the range $150-250 \mu \mathrm{m}$, carefully purified according to the procedures described in detail by Kreutzer et al. (in prep.), annealed $\left(30 \mathrm{~min}\right.$ at $490{ }^{\circ} \mathrm{C}$ ), homogenised and distributed among the eight laboratories. Further information on geological origin as well as secondary electron microscopy data and basic luminescence characterisation of this sample are summarised in Kreutzer et al. (in prep.).

160

\subsection{Measurement setup and data evaluation}

For interlaboratory comparison, we used seven Risø TL/OSL DA-15 and DA-20, two Freiberg Instruments lexsyg research luminescence readers as well as one lexsyg smart reader

165 (Bøtter-Jensen et al., 2010; Richter et al., 2013, 2015), as specified in Table 2. In terms of sample carriers and laboratory-specific procedures of aliquot preparation, we kept the individual protocols of each laboratory (Table 2) to monitor the influence of these parameters on the determined $E$ and $s$ parameters. Data reduction, calculation of the kinetic parameters and plotting were carried out in a standardised way for all laboratories using a self-written $\mathbf{R}$

170 (R Development Core Team, 2017) script and the packages 'Luminescence' (Kreutzer et al., 2012; Kreutzer et al., 2017) and 'ggplot2' (Wickham, 2009).

\section{Table 2}


175 The basic principles of the different methods we used to constrain $E$ and $s$ for the reference sample are reviewed briefly in the following sections. Specific measurement parameters relevant for the interlaboratory comparison are given in Sections 4.2.1, 4.2.2 and 4.2.3.

\subsection{Isothermal decay method}

180

The lifetime of a luminescence signal can be measured directly by holding the sample at a fixed temperature for a prolonged time while recording the emitted phosphorescence (isothermal TL). For first-order kinetics, the phosphorescence intensity $I$ as a function of time $t$ can be written as

185

$$
I(t)=I_{0} \cdot e^{-\frac{t}{\tau}}
$$

with $I_{0}$ being the phosphorescence intensity at $t=0$ and $\tau$ again being the lifetime as defined in Eq. (1). In a graph of $\ln (I)$ against isothermal holding time, the slope of the straight line hence represents $-\tau^{-1}$. Taking the logarithm of the inverse of Eq. (1) gives

$$
\ln \tau^{-1}=\ln s-\frac{E}{k_{B} T}
$$

195

If we conduct a series of isothermal TL measurements at different temperatures and create a plot of $\ln \tau^{-1}$ against $1 / k_{\mathrm{B}} T$, the slope of the straight line then corresponds to $-E$. The intercept of this line with the y-axis equals $\ln s$, from which $s$ can be easily obtained (Chen and McKeever, 1997).

\subsection{Initial rise method}

The initial rise (IR) method, first introduced by Garlick and Gibson (1948), is based on the approximation that the initial luminescence intensity of a TL peak is proportional to exp($\left.E / k_{\mathrm{B}} T\right)$, no matter what the order of kinetics is. This relation holds up to a temperature corresponding to an intensity of $\sim 10-15 \%$ of the maximum peak intensity, in a temperature range in which the variation of the electron population in the trap can be considered 
negligible, however depending on the lower limit of the temperature window considered for data evaluation (Pagonis et al., 2006). The thermal activation energy $E$ can thus be obtained from the slope in the plot of $\ln I$ against $1 / k_{\mathrm{B}} T$ :

$$
\ln I=\ln C-\frac{E}{k_{B} T}
$$

215 with $I$ being the TL intensity and $C$ a constant. Once $E$ has been determined, $s$ can be calculated using the following formula (Mahesh et al., 1989):

$$
s=\frac{q E}{k_{B} T_{m}^{2}} e^{\frac{E}{k_{B} T_{m}}}
$$

where $q$ is the heating rate (in $\mathrm{K} \mathrm{s}^{-1}$ ) and $T_{\mathrm{m}}$ is the temperature at maximum peak intensity. The IR method, however, may yield erroneous results for TL peaks affected by thermal quenching (Aitken, 1985; Chen and Pagonis, 2011). Investigations by Wintle (1975) and Schilles et al. (2001) suggest that thermal quenching begins at temperatures $>100-120{ }^{\circ} \mathrm{C}$, so that we assume our results are not significantly affected (upper limit of temperature window $\left.<100{ }^{\circ} \mathrm{C}\right)$.

\subsection{Hoogenstraaten method}

230 In the Hoogenstraaten approach (Hoogenstraaten, 1958), the TL glow curve is recorded using different heating rates $q$ (in $\mathrm{K} \mathrm{s}^{-1}$ ), which should cover several decades. The slope of fitted data points in a plot of $\ln \left(T_{\mathrm{m}}{ }^{2} / q\right)$ against $1 / k_{\mathrm{B}} T_{\mathrm{m}}$ provides $E$. The frequency factor $s$ is then derived from the following formula:

$$
s=(E-W) \cdot e^{\frac{p}{k_{B}}}
$$

where $W$ is the quenching energy (in eV), which is assumed to be zero for the $110^{\circ} \mathrm{C}$ TL peak (see Section 3.4), and $p$ is the intercept of the fit with the y-axis in the Hoogenstraaten plot (Spooner and Franklin, 2002). This method thus exploits the fact that $T_{\mathrm{m}}$ increases with $q$ 
(independent of any thermal lag effects) and is only applicable to peaks obeying first-order kinetics.

\section{Results}

\subsection{Thermal lag}

As outlined in Section 1, thermal lag should be minimised for measurements utilised for trap parameter estimation. Increasing heating rates are known to shift the TL peak corresponding to a specific type of trap towards higher glow curve temperatures, which is on the one hand a basic feature of TL, but on the other hand due to a rising difference between target sample temperature and actual sample temperature. This is shown in Fig. 1 for the FB quartz sample, where the $110^{\circ} \mathrm{C}$ TL peak was measured after regenerative $\beta$-irradiation of $1 \mathrm{~Gy}$. It moves from $\sim 50{ }^{\circ} \mathrm{C}$ glow curve temperature for a heating rate of $0.1 \mathrm{~K} \mathrm{~s}^{-1}$ to $\sim 80-140{ }^{\circ} \mathrm{C}$ for $5 \mathrm{~K} \mathrm{~s}^{-1}$. We chose $0.1 \mathrm{~K} \mathrm{~s}^{-1}$ as the lowest heating rate to display since we are not sure about accurate temperature control at even lower heating rates. The initial signal decay at low heating rates is most pronounced for measurements performed on lexsyg readers and may represent phosphorescence, i.e. isothermal depletion of the $110{ }^{\circ} \mathrm{C}$ TL trap or even shallower traps (e.g., Schlesinger, 1964; Jani et al., 1983). The TL peak temperatures $\left(T_{\mathrm{m}}\right)$ as a function of all employed heating rates $\left(0.02,0.05,0.1,0.2,0.5,1,2,3,4,5 \mathrm{~K} \mathrm{~s}^{-1}\right)$ are shown for all reader

260 setups in the supplementary materials.

While for low heating rates $\left(<0.1 \mathrm{~K} \mathrm{~s}^{-1}\right)$, varying thermal contact, thermal inertia and poor temperature reproducibility of the heating systems between readers result in TL peak temperature deviations of $\sim 10 \mathrm{~K}$ between laboratories, they increase up to $\sim 60 \mathrm{~K}$ for a heating rate of $5 \mathrm{~K} \mathrm{~s}^{-1}$ (Fig. 1). Reader setup 267 shows the highest thermal lag (or, as a consequence, underestimation of heating rate) at $5 \mathrm{~K} \mathrm{~s}^{-1}\left(T_{\mathrm{m}}=143 \pm 6{ }^{\circ} \mathrm{C} ; n=5 ; n\right.$ being the number of analysed TL glow curves), setup 154 the smallest $\left(T_{\mathrm{m}}=82 \pm 1{ }^{\circ} \mathrm{C} ; n=3\right)$. When combining the TL peak temperatures for different laboratories with the data on sample carriers (Table 2), only a weak correlation between sample carrier thickness and the observed peak temperature emerges $(r=0.44)$. Hence, beside the thermal inertia of the sample carriers, other factors such as their shape (influencing thermal contact), the amount of silicone oil used to fix the grains on the carrier or the temperature calibration of the thermocouple may contribute to the observed interlaboratory variations. Considering the discrepancies in TL peak temperature of up to $\sim 18 \mathrm{~K}$ for several aliquots measured in the same laboratory (at $5 \mathrm{~K} \mathrm{~s}^{-1}$; e.g., reader setup 
374 as an extreme case, see Fig. 2), it appears that the thermal contact between heater and sample carrier is the main determining factor limiting intra-laboratory reproducibility.

\section{Figure 1 [1 column]}

Figure 2 [1 column]

\subsection{Kinetic parameters $E$ and $s$}

The results for the three methods employed to determine $E$ and $s$ for the FB quartz are given in full in the supplementary materials; representative plots are shown below in the respective sections.

\subsubsection{Isothermal decay method}

To determine the thermal activation energy $E$ and the frequency factor $s$ by means of the isothermal decay method, five aliquots of the FB quartz were repeatedly $\beta$-irradiated with a dose of $1 \mathrm{~Gy}$ and subsequently held at temperatures in the range of $30-80{ }^{\circ} \mathrm{C}$ (in $10^{\circ} \mathrm{C}$ increments) while recording the phosphorescence (= isothermal TL; Fig. 3a). After the phosphorescence measurements, the sample was heated to $160{ }^{\circ} \mathrm{C}$ to completely empty the $110^{\circ} \mathrm{C}$ TL trap prior to the next regeneration cycle. Due to incomplete thermal stabilisation (thermal lag) at the beginning of the phosphorescence curve we observed an initial peak structure for most of the decay curves (see Fig. 3a) which was discarded for further analyses (the number of removed channels varied from setup to setup). The result is a straight line in a plot of $\ln$ (phosphorescence) versus time from which the lifetime was extracted by curve fitting (Eq. 2). The natural logarithm of the inverse lifetime derived from the decay curves for each isothermal holding temperature was then plotted against $1 / k_{\mathrm{B}} T$ (Fig. $3 \mathrm{~b}$ ) and $E$ and $s$ parameters were determined as described in Section 3.3.

\section{Figure 3 [2 columns]}

$E$ values obtained from the isothermal decay analyses span the range from $0.73 \pm 0.01 \mathrm{eV}$ 305 (reader 150) to $1.02 \pm 0.01 \mathrm{eV}$ (reader 12-re-01-0007), while the corresponding $s$ values lie between $1.20 \pm 0.34 \times 10^{9} \mathrm{~s}^{-1}$ (reader 150) and 7.28 $\pm 1.72 \times 10^{13} \mathrm{~s}^{-1}$ (reader 12-re-01-0007) (Fig. 4 and Table 3). While thermal activation energies vary by $\sim 40 \%$ (Fig. $4 \mathrm{a}$ ) and frequency 
factors by a factor of $7 \times 10^{4}$ (Fig. 4b) between laboratories, resulting lifetimes at $20{ }^{\circ} \mathrm{C}$ differ by $\sim 86 \%$ at maximum, although consistent within uncertainties throughout (Fig. 4c).

\section{Table 3}

\section{Figure 4 [1 column]}

\subsubsection{Initial rise method}

Application of the IR method for $E$ and $s$ determination was hampered by the presence of strong phosphorescence in the low-temperature range of the glow curve (cf. Fig. 1), especially in case of low heating rates $\left(<1 \mathrm{~K} \mathrm{~s}^{-1}\right)$. This problem persisted even after inserting a pause of $600 \mathrm{~s}$ after $\beta$-irradiation ( $1 \mathrm{~Gy}$ ) prior to TL measurement. These conditions do not allow for an accurate evaluation of the kinetic parameters and we, therefore, restricted our analyses to TL glow curves recorded with heating rates of $0.5,1,2$ and $3 \mathrm{~K} \mathrm{~s}^{-1}$. Higher heating rates were omitted due to intense thermal lag (see Section 4.1 and Fig. 1). The temperature range of the glow curve used for IR evaluation was chosen such that the data points in a plot of $\ln I$ against $1 / k_{\mathrm{B}} T$ yield a straight line with the upper limit defined by the temperature corresponding to $15 \%$ of the maximum peak intensity. For the adopted heating rates, the lower limits are $24{ }^{\circ} \mathrm{C}$ $\left(0.5 \mathrm{~K} \mathrm{~s}^{-1}\right.$ and $\left.1 \mathrm{~K} \mathrm{~s}^{-1}\right), 30{ }^{\circ} \mathrm{C}\left(2 \mathrm{~K} \mathrm{~s}^{-1}\right)$ and $34{ }^{\circ} \mathrm{C}\left(3 \mathrm{~K} \mathrm{~s}^{-1}\right)$.

Evaluation results $\left(E, s\right.$ and $\tau\left(\right.$ at $\left.20{ }^{\circ} \mathrm{C}\right)$ ) for the IR method are shown as boxplots in Fig. 5. A comprehensive table containing the numerical results can be found in the supplementary materials. Determined thermal activation energies range from $0.67 \pm 0.04 \mathrm{eV}$ (reader 14-1601-0008; for $\left.0.5 \mathrm{~K} \mathrm{~s}^{-1}\right)$ to $1.25 \pm 0.05 \mathrm{eV}$ (11-std-01-0001; for $\left.0.5 \mathrm{~K} \mathrm{~s}^{-1}\right)$ with the largest variation observed for a heating rate of $0.5 \mathrm{~K} \mathrm{~s}^{-1}$, possibly due to phosphorescence still contributing to the initial TL signal. For $3 \mathrm{~K} \mathrm{~s}^{-1}$, the range narrows down to $\sim 0.75 \mathrm{eV}$ to $\sim 1.05$ $\mathrm{eV}$. Frequency factors derived from the IR method cover the range $2.8 \pm 2.1 \times 10^{8} \mathrm{~s}^{-1}$ (reader 14-16-01-0008; for $1 \mathrm{~K} \mathrm{~s}^{-1}$ ) to $8.4 \pm 14 \times 10^{17} \mathrm{~s}^{-1}$ (11-std-01-0001; for $0.5 \mathrm{~K} \mathrm{~s}^{-1}$ ). For low heating rates, the measurements conducted on lexsyg systems (14-16-01-0008 and 11-std-01$0001)$ yield a large spread of $E$ and $s$ values for the five measured aliquots, compared to those carried out on Risø readers. It is further worth noting that the data produced with lexsyg readers bracket the $E$ and $s$ values resulting from measurement on Risø machines for the heating rates 0.5 and $1 \mathrm{~K} \mathrm{~s}^{-1}$, while the lexsyg results from readers 14-16-01-0008 and 11-std01-0001 converge for higher heating rates. The same applies to the $s$ values, since $s$ is directly 
derived from $E$ (see Section 3.4). Calculated lifetimes $\tau$ at $20{ }^{\circ} \mathrm{C}$ vary from $20.0 \pm 1.3 \mathrm{~min}$ (14-16-01-0008; for $\left.1 \mathrm{~K} \mathrm{~s}^{-1}\right)$ to $267 \pm 11 \mathrm{~min}\left(267\right.$; for $\left.3 \mathrm{~K} \mathrm{~s}^{-1}\right)$, thus fluctuating by an order of magnitude. However, the majority of data points lies between 50 and $100 \mathrm{~min}$, which is consistent with direct measurements of $\tau$ at room temperature $(50.4 \pm 0.9 \mathrm{~min}$; Vaccaro et al., 2017 ) by means of isothermal TL. In contrast to the calculated $E$ and $s$ values, the lifetimes appear to show increasing variability with growing heating rate.

\section{Figure 5 [1 column]}

\subsubsection{Hoogenstraaten method}

To obtain kinetic parameters using the Hoogenstraaten method, the $110^{\circ} \mathrm{C}$ TL peak was measured repeatedly after $\beta$-irradiation of 1 Gy employing heating rates of $0.02,0.05,0.1$, $0.2,0.5,1,2,3,4$ and $5 \mathrm{~K} \mathrm{~s}^{-1}$. Then, the temperature of the TL peak maximum $\left(T_{\mathrm{m}}\right)$ was extracted and a Hoogenstraaten plot constructed (Fig. 6), from which the slope corresponds to $E$; $s$ was calculated according to the formula given in Section 3.5. However, due to increased thermal lag and enhanced spread of $T_{\mathrm{m}}$ values for heating rates $>1 \mathrm{~K} \mathrm{~s}^{-1}$, we considered only data points generated by heating rates in the range $0.02-1 \mathrm{~K} \mathrm{~s}^{-1}$ for the Hoogenstraaten plots.

\section{Figure 6 [1 column]}

Resulting kinetic parameters for the Hoogenstraaten method are summarised in Table 3. Extreme values for $E$ and $s$ are $0.52 \pm 0.02 \mathrm{eV}$ (reader 267) and $1.08 \pm 0.01 \mathrm{eV}$ (reader 154), and $5.1 \pm 3.1 \times 10^{5} \mathrm{~s}^{-1}$ (reader 14-16-01-0008) and $9.7 \pm 2.5 \times 10^{14} \mathrm{~s}^{-1}$ (reader 154); these parameters thus fluctuate by factors of $\sim 2$ and $10^{9}$, respectively. If data points resulting from larger heating rates $\left(>1 \mathrm{~K} \mathrm{~s}^{-1}\right)$ would be taken into account for analysis of kinetic parameters, $E$ values would be slightly lower $(0.74 \pm 0.15 \mathrm{eV}$; average of all laboratories $)$ than $E$ values resulting exclusively from lower heating rates $(0.83 \pm 0.14 \mathrm{eV}$; Table 4$)$, although indistinguishable within uncertainties. Calculated lifetimes $\tau$ range between $22 \pm 17 \mathrm{~min}$ (reader 14-16-01-0008) and $57 \pm 22 \mathrm{~min}$ (reader 154).

\section{Figure 7 [1 column]}


For the $110{ }^{\circ} \mathrm{C}$ TL peak, the lifetime $\tau$ can be determined directly by recording the emitted phosphorescence at room temperature $\left(\sim 20^{\circ} \mathrm{C}\right)$ after artificial irradiation (1 Gy). Fitting Eq. 2 to the decay curve provides $\tau$. For the FB reference quartz sample, the lifetime was determined both on a Risø (150) and a lexsyg research (11-std-01-0001) reader by fitting Eq. (2) to the phosphorescence decay curve, yielding values of $69.0 \pm 0.4$ and $70.1 \pm 0.3 \mathrm{~min}$, respectively (Fig. 8). It has to be noted that the first $10 \mathrm{~s}$ of the decay curve had to be removed due to a rapidly decreasing phosphorescence component of unknown origin. Additionally, it is unclear whether the target measurement temperature of $20^{\circ} \mathrm{C}$ could be maintained in all experiments due to differences in room temperature between measurements and the lost heat of the luminescence readers.

\section{Figure 8 [1 column]}

\subsection{Summary of results}

Arithmetic average values for $E$ and geometric average values for $s$ (due to the lognormal distribution of $s$ values for the isothermal decay and Hoogenstraaten methods) across all laboratories as obtained for the three applied methods are given in Table 4. The relative standard deviation ( $1 \sigma$; rel. SD) of the averaged activation energies amounts to $\sim 9-20 \%$, and within these uncertainties all mean values for $E$ are consistent with each other. Results from the isothermal decay and IR methods range between $0.87 \pm 0.12$ and $0.9 \pm 0.2 \mathrm{eV}$, respectively; the Hoogenstraaten method yields a slightly, but not significantly, lower $E$ value of $0.83 \pm 0.14 \mathrm{eV}$. Averaged values for $s$ are in the order of $10^{11}-10^{12} \mathrm{~s}^{-1}$. Lifetimes $\tau$ calculated with these average $E$ and $s$ values vary between $\sim 40$ and $\sim 70 \mathrm{~min}$ (IR method at low and high heating rates). By contrast, lifetimes $\tau_{\mathrm{s}}$ resulting from averaging the individual $\tau$ values obtained for each laboratory are consistently higher for the IR method than the $\tau$ values derived from averaged $E$ and $s$ values. This can be explained with the fact that the distribution of $s$ values produced with this method is not lognormal, what in turn influences the geometric 405 mean. Both the average value and the standard deviation of $\tau_{\mathrm{s}}$ rise with increasing heating rate employed in IR experiments. The rel. SD reflects the variation in $E$ and $s$ values among laboratories; thus, the isothermal decay method yields the most reproducible results (rel. SD $\sim 16 \%$ ), whereas high heating rates used for IR measurements produce increased scatter (rel. SD up to $\sim 50 \%$ ). 


\section{Table 4}

\section{Discussion}

\subsection{Thermal lag}

Comparison of $\mathrm{TL}$ glow curves of the $110^{\circ} \mathrm{C} \mathrm{TL}$ peak of the FB reference quartz sample recorded at different heating rates demonstrated large variations of the actual sample temperature in relation to the target sample temperature (thermal lag). For the measurement equipment used in this comparative study, we detected temperature differences of $\sim 40{ }^{\circ} \mathrm{C}$ between laboratories for high heating rates (e.g., reader setups 154 and 267; Fig. 1). For the more likely case that different sample holders and aliquot preparation routines are used, temperature differences may exceed $60^{\circ} \mathrm{C}$ for the $110^{\circ} \mathrm{C}$ TL peak and further increase for higher glow curve temperatures (Kitis et al., 2015). These observations imply that accurate temperature control of the sample and reproducibility of TL glow curves using standard luminescence dating equipment cannot be taken for granted. When aiming at measuring kinetic parameters of a specific type of electron trap, it should therefore be of paramount importance to minimise thermal lag effects and to calibrate the heating element accurately. In case a method based on varying heating rates is used to derive kinetic parameters, the heating rate should be reduced $\left(<1 \mathrm{~K} \mathrm{~s}^{-1}\right)$. Furthermore, it appears advisable to increase the thermal contact between the temperature sensor (and heating element) and sample by employing thinner sample carriers of high thermal conductivity. Powdered sample material on aluminium cups is reported to cause smaller thermal lag compared to sand-sized grains fixed with silicone oil on stainless steel discs (Jain et al., 2007). Apart from reading off the (relative) amount of thermal lag from the difference in TL peak temperatures, Jain et al. (2007) suggest to use the structure and time of occurrence of the peak often observed at the beginning of isothermal TL measurements to estimate the amount of thermal lag (cf. Fig. 3a). By conducting separate measurements, it is further possible to quantify and correct for thermal lag, as proposed by Kitis and Tuyn (1998, 1999) and Kitis et al. (2015), but this is outside the scope of the present investigation.

\subsection{Kinetic parameters and their variability among various methods and laboratories}


The relative uncertainties of individual kinetic parameters obtained in both previous studies and the present investigation ( $E: \sim 1-3 \%, s: \sim 12-80 \%$; cf. Tables 1 and 3 ) are much smaller than the spread of $E$ and $s$ values among various laboratories (Table 4). This finding implies that the accuracy with which these absolute physical parameters can be measured is assumed to be higher than it actually is. In other words, the systematic error associated with a single value of $E$ or $s$ determined with the setup in one specific laboratory or with one specific

450 luminescence reader is likely to be underestimated, as long as the problems of thermal lag and accurate temperature control have to been addressed rigorously. Nevertheless, despite the large variation of laboratory-specific $E$ and $s$ values, the average values for each method are statistically indistinguishable from each other based on the (quite large) associated standard deviation. The isothermal decay and the IR methods produce very similar results for $E$ and $s$ (with the latter covering the same order of magnitude), indicating that averaging effects cancel out laboratory-specific thermal lag and/or deficiencies in sample temperature control. Thermal lag is likely to be the main reason for the Hoogenstraaten method to give comparatively low $E$ values and lifetimes. Especially the data points in the Hoogenstraaten plot resulting from high heating rates $\left(>3 \mathrm{~K} \mathrm{~s}^{-1}\right)$ show substantial deviation from the linear fit to the remaining data points associated with lower heating rates (Fig. 6). The fit to the full dataset hence yields a lower slope corresponding to a smaller $E$ value. Consequently, employing small heating rates should be mandatory to reduce systematic errors in experimentally derived kinetic trapping parameters. The present dataset demonstrates that it is very challenging to find accurate absolute physical trapping parameters with standard

465 luminescence dating equipment, at least without applying correction procedures for thermal lag. Even for low heating rates (entailing the lowest possible thermal lag) and methods not depending on heating rate (isothermal decay; temperature accuracy required only in the lowtemperature range $<80^{\circ} \mathrm{C}$ ) we observed variations in $E$ and $s$ among the contributing laboratories exceeding the individual uncertainties.

470 The standard deviation of a selection of published values on the thermal activation energy $E$ of the $110^{\circ} \mathrm{C}$ TL peak (i.e., $0.14 \mathrm{eV}$; Table 1 ) is of comparable magnitude to that of our determined $E$ values (Table 4 ). The variation in physical trap parameters is thus not restricted to the technical setups used in this study, but occurs also for other experimental arrangements. These considerations are based on the premise that there is just one single type of trap giving rise to the $110^{\circ} \mathrm{C}$ TL signal. However, at least for annealed synthetic quartz, it was shown that the $110^{\circ} \mathrm{C}$ TL peak might be composed of more than one component (Petrov and Bailiff, 1995). 
The main purpose for determining the kinetic parameters $E$ and $s$ for a specific trap is to estimate its electron retention time (lifetime) and hence its thermal stability at a certain temperature in the context of dosimetry and dating. Since $E$ and $s$ influence the lifetime in the opposite way, it is more meaningful to this aim to compare the lifetimes obtained by the three methods applied with those determined directly (by measuring phosphorescence at a specific temperature). This direct comparison is of course not feasible for signals with lifetimes exceeding several hours (unless measured at elevated temperatures). The lifetimes measured directly $(69.0 \pm 0.4$ and $70.1 \pm 0.3 \mathrm{~min}$ for Risø and lexsyg research readers; $50 \pm 1 \mathrm{~min}$, as determined by Vaccaro et al., 2017, however for a different quartz sample with different thermal treatment) match most closely with results from the isothermal decay method $\left(\tau_{\mathrm{s}}=57\right.$ $\pm 9 \mathrm{~min}$ ) and the IR method at low heating rates (Table 4). Employing higher heating rates produces systematically increasing lifetimes for the latter approach, as is consistent with the observation of enlarged thermal lag for such heating rates. The same explanation applies to the lifetimes extracted from Hoogenstraaten plots, here leading to underestimated values. When comparing the isothermal decay results of Risø and lexsyg readers (Figs. 4 and 5; Table 3 ), it is striking that the latter yield both $E$ and $s$ values consistently and significantly larger than values obtained with Risø readers (while resulting lifetimes at room temperature are statistically identical for both systems). Therefore, it appears that the design of the measurement apparatus (particularly the heating element) and the temperature calibration have significant influence on the absolute quantities $E$ and $s$ derived by this method. The IR method performed on the lexsyg smart (14-16-01-0008) and research (11-std-01-0001) readers returns kinetic parameters at low heating rates $\left(<2 \mathrm{~K} \mathrm{~s}^{-1}\right)$ at the lower and upper range, i.e. extreme values, which again underpins the effect of the luminescence measurement equipment on trapping parameters.

\subsection{Implications for dosimetry, dating and thermochronometry}

505 In order to calculate the lifetime of a specific luminescence signal in the context of dosimetry, dating and thermochronometry, kinetic parameters, which are determined in comparatively short time periods via laboratory experiments, are extrapolated to geological periods. Because of this extrapolation and the Arrhenius term in Eq. (1), small systematic errors in the parameters strongly affect the estimated lifetime on long timescales. This effect is particularly pronounced for thermochronometric application of luminescence signals, where the 
temperature-sensitivity of the relative occupancy level of an electron trap is exploited to deduce potential changes in past ambient/subsurface temperatures.

The results of this comparative study have shown that depending on the equipment and laboratory routines, we are looking at slightly different TL and OSL signals (if the signal is sourced from more than one trap), according to the difference between actual and target temperature during measurement. Since the measured equivalent dose $\left(D_{\mathrm{e}}\right)$ is a temperatureor time-integrated quantity (TL and OSL, respectively), it is not correlated with the lifetime determined for the respective signal (provided the lifetime is sufficiently long in relation to the dating period), which itself is based on the change of this signal as a function of temperature or heating rate. If we assume that the actual temperature lags behind the target temperature by a certain amount, then the determined lifetime of the recorded signal will be erroneously high. The consequence is an underestimated dose or age derived from a luminescence signal thought to have a thermal stability higher than it actually is. Accurate temperature control achieved by thorough calibration of heating elements and

525 reducing thermal lag as much as possible thus represents the key to measuring valid kinetic parameters of a specific luminescence signal.

\section{Conclusions}

530 The interlaboratory comparison and the analysis of the derived dataset of $E$ and $s$ values for the $110^{\circ} \mathrm{C}$ TL peak of the Fontainebleau quartz reference sample allows drawing the following conclusions:

- There is considerable thermal offset between actual and target sample temperature for $\mathrm{TL}$ measurements among laboratories that can reach up to $60^{\circ} \mathrm{C}$ for the $110^{\circ} \mathrm{C} \mathrm{TL}$ peak in quartz at high heating rates $\left(>3 \mathrm{~K} \mathrm{~s}^{-1}\right)$.

- $E$ and $s$ values for the $110^{\circ} \mathrm{C}$ TL peak were determined with the isothermal decay, the initial rise and the Hoogenstraaten methods and span the range $\sim 0.5-1.2 \mathrm{eV}$ and $5 \mathrm{x}$ $10^{5}-1 \times 10^{18} \mathrm{~s}^{-1}$, respectively.

540 - For the equipment used in this study, individual uncertainties on $E$ and $s$ values significantly underestimate the systematic errors introduced by laboratory-specific measurement equipment/routines, i.e., we observed an unexpected overdispersion in the $E$ and $s$ parameters. 
- Averaged across laboratories, all three methods produce consistent results for $E$ within $1 \sigma$ standard deviation between $0.83 \pm 0.14 \mathrm{eV}$ (Hoogenstraaten) and $0.90 \pm 0.17 \mathrm{eV}$ (initial rise at $0.5 \mathrm{~K} \mathrm{~s}^{-1}$ ). The less dispersed results were obtained with the isothermal decay method. Lifetimes derived from the isothermal decay and the initial rise method (at low heating rate) conform best to the directly measured value.

- Accurate control of sample temperature is essential to reduce these systematic uncertainties and might be improved by regularly calibrating the heating element as well as by lowering the heating rates and using thin sample carriers of high thermal conductivity along with small grain sizes of the target material.

- Temperature offset causes erroneously high lifetimes with the possible consequence of dose or age underestimation.

\section{Acknowledgements}

We acknowledge the support of the Bavarian Research Alliance (BayFor) for financing the project meeting in Bayreuth (BayIntAn_UBT_2016_74). The work of SK was financed by a programme supported by the ANR - n॰ ANR-10-LABX-52, that of JF by the DFG (project "Modelling quartz luminescence signal dynamics relevant for dating and dosimetry", SCHM 3051/4-1).

\section{Supplementary material}

This article offers supplementary HTML files containing

(1) Plots of TL glow curves up to $160^{\circ} \mathrm{C}$ for heating rates of $0.02,0.05,0.1,0.2,0.5,1,2$, 3,4 and $5 \mathrm{~K} \mathrm{~s}^{-1}$ for all aliquots measured in all laboratories, both as 'absolute' and normalised curves,

(2) Glow curve fitting with a first-order kinetics function for curves from all laboratories,

(3) A comparison of $110^{\circ} \mathrm{C}$ TL peak positions for all laboratories as a function of heating rate,

(4) Evaluation results and plots for the isothermal decay method for all laboratories,

(5) Numerical results and plots of the IR method for all laboratories.

Additionally, all raw measurement data (.seq and .bin-files) of the interlaboratory comparison are available at the EarthChem Library database at: 
http://www.earthchem.org/library/browse/view?id=1095

doi:10.1594/IEDA/100711

Readers are highly encouraged to further explore the data with regard to optimising kinetic parameter analysis and to compare them to own data. 


\section{References}

Aitken, M., 1985. Thermoluminescence dating. Academic Press, London.

590

Bailiff, I., 1994. The pre-dose technique. Radiation Measurements 23, 471-479.

Bailiff, I., Haskell, E., 1983. The Use of the Pre-Dose Technique for Environmental Dosimetry. Radiation Protection Dosimetry 6, 245-248.

595

Bøtter-Jensen, L., Thomsen, K.J., Jain, M., 2010. Review of optically stimulated luminescence (OSL) instrumental developments for retrospective dosimetry. Radiation Measurements 45, 253-257.

600 Chen, R., McKeever, S., 1997. Theory of Thermoluminescence and Related Phenomena. World Scientific, Singapore.

Chen, G., Li, S.-H., Murray, A.S., 2000. Study of the $110^{\circ} \mathrm{C}$ TL peak sensitivity in optical dating of quartz. Radiation Measurements 32, 641-645.

605

Chen, R., Pagonis, V., 2011. Thermally and Optically Stimulated Luminescence - A Simulation Approach. Wiley, West Sussex.

Fleming, S., 1973. The pre-dose technique: A new thermoluminescence dating method. Archaeometry 15, 13-30.

Fleming, S., 1979. Thermoluminescence Techniques in Archaeology. Clarendon Press, Oxford.

615 Garlick, G., Gibson, A., 1948. The electron trap mechanism of luminescence in sulphide and silicate phosphors. Proceedings of the Physical Society 60, 574-590.

Godfrey-Smith, D.I., Ilani, S., 2004. Past thermal history of goethite and hematite fragments from Qafzeh Cave deduced from thermal activation characteristics of the $110^{\circ} \mathrm{C}$ TL peak of enclosed quartz grains. Revue d'Archéométrie 28, 185-190. 
Göksu, H.Y., Weiser, A., Regulla, D.F., 1989. $110^{\circ} \mathrm{C}$ TL peak records the ancient heat treatment of flint. Ancient TL 7, 15-17.

625 Guralnik, B., Jain, M., Herman, F., Ankjærgaard, C., Murray, A.S., Valla, P.G., Preusser, F., King, G.E., Chen, R., Lowick, S.E., Kook, M., Rhodes, E.J., 2015. OSLthermochronometry of feldspar from the KTB borehole, Germany. Earth and Planetary Science Letters 423, 232-243.

630 Herman, F., Rhodes, E.J., Braun, J., Heiniger, L., 2010. Uniform erosion rates and relief amplitude during glacial cycles in the Southern Alps of New Zealand, as revealed from OSL-thermochronology. Earth and Planetary Science Letters 297, 183-189.

Hoogenstraaten, W., 1958. Electron traps in zinc-sulphide phosphors. Philips Research 635 Reports 13, 515-693.

Huntley, D., 2006. An explanation of the power-law decay of luminescence. Journal of Physics: Condensed Matter 18, 1359-1365.

640 Huntley, D.J., Lamothe, M., 2001. Ubiquity of anomalous fading in K-feldspars and the measurement and correction for it in optical dating. Canadian Journal of Earth Sciences 38, 1093-1106.

Huntley, D., Short, M., Dunphy, K., 1996. Deep traps in quartz and their use for optical dating 645 Canadian Journal of Physics 74, 81-91.

Jain, M., Murray, A.S., Bøtter-Jensen, L., 2003. Characterisation of blue-light stimulated luminescence components in different quartz samples: implications for dose measurement. Radiation Measurements 37, 441-449.

650

Jain, M., Bøtter-Jensen, L., Murray, A.S., Essery, R., 2007. A peak structure in isothermal luminescence signals in quartz: Origin and implications. Journal of Luminescence 127, 678-688. 
Jani, M.G., Halliburton, L.E., Kohnke, E.E., 1983. Point defects in crystalline $\mathrm{SiO}_{2}$ :

Thermally stimulated luminescence above room temperature. Journal of Applied Physics $54,6321-6328$.

Kaya Keles, S., Meriç, N., Polymeris, G.S., 2016. Dose response on the $110^{\circ} \mathrm{C}$ thermoluminescence peak of un-heated, synthetic Merck quartz. Physica B 493, 17-24.

King, G.E., Herman, F., Lambert, R., Valla, P., Guralnik, B., 2016. Multi-OSLthermochronometry of feldspar. Quaternary Geochronology 33, 76-87.

665 King, G.E., Herman, F., Guralnik, B., 2016. Northward migration of the eastern Himalayan syntaxis revealed by OSL thermochronometry. Science 353, 800-804.

King, G.E., Guralnik, B., Valla, P.G., Herman, F., 2016. Trapped-charge thermochronometry and thermometry: A status review. Chemical Geology 446, 3-17.

670

Kitis, G., Tuyn, J., 1998. A simple method to correct for the temperature lag in TL glowcurve measurements. Journal of Physics D: Applied Physics 31, 2065-2073.

Kitis, G., Tuyn, J., 1999. Correction for Temperature Lag and Thermal Gradient Effects Arising During Thermoluminescence Readout. Radiation Protection Dosimetry 84, 371374.

Kitis, G., Kiyak, N.G., Polymeris, G.S., 2015. Temperature lags of luminescence measurements in a commercial luminescence reader. Nuclear Instruments and Methods in Physics Research B 359, 60-63.

Kreutzer, S., Schmidt, C., Fuchs, M.C., Dietze, M., Fischer, M., Fuchs, M., 2012. Introducing an R package for luminescence dating analysis. Ancient TL 30, 1-8. 2017. Luminescence: Comprehensive Luminescence Dating Data Analysis. R package, version 0.7.5. https://CRAN.R-project.org/package=Luminescence 
Koul, D., Singhvi, A., Nambi, K., Bhat, C., Gupta, P., 1996. Feasibility of estimating firing temperature using the $110^{\circ} \mathrm{C}$ TL peak of quartz. Applied Radiation and Isotopes 47, 191194.

Mahesh, K., Weng, P., Furetta, C., 1989. Thermoluminescence in solids and its applications. Nuclear Technology Publishing, Ashford.

695

Murray, A.S., Buylaert, J.-P., Thiel, C., 2015. A luminescence dating intercomparison based on a Danish beach-ridge sand. Radiation Measurements 81, 32-38.

Pagonis, V., Tatsis, E., Kitis, G., Drupieski, C., 2002. Search for Common Characteristics in the Glow Curves of Quartz of Various Origins. Radiation Protection Dosimetry 100, 373376.

Pagonis, V., Kitis, G., Chen, R., 2003. Applicability of the Zimmerman predose model in the thermoluminescence of predosed and annealed synthetic quartz samples. Radiation Measurements 37, 267-274.

Pagonis, V., Kitis, G., Furetta, C., 2006. Numerical and Practical Exercises in Thermoluminescence. Springer, New York.

710 Petrov, S., Bailiff, I., 1995. The $110^{\circ} \mathrm{C}$ TL peak in synthetic quartz. Radiation Measurements 24, 519-523.

Preusser, F., Chithambo, M.L., Götte, T., Martini, M., Ramseyer, K., Sendezera, E.J., Susino, G.J., Wintle, A.G., 2009. Quartz as a natural luminescence dosimeter. Earth-Science Reviews 97, 184-214.

Richter, D., Richter, A., Dornich, K., 2013. Lexsyg - A new system for luminescence research Geochronometria 40, 220-228.

720 Richter, D., Richter, A., Dornich, K., 2015. Lexsyg smart — a luminescence detection system for dosimetry, material research and dating application. Geochronometria 42, 202-209. 
R Development Core Team, 2017. R: A Language and Environment for Statistical Computing. R Foundation for Statistical Computing, Vienna, Austria. http://r-project.org 725

Schlesinger, M., 1964. Thermoluminescence in aluminium-containing quartz. Physics Letters $10,49-50$.

Schmidt, C., Friedrich, J., Zöller, L., 2015. Thermochronometry using red TL of quartz? Numerical simulation and observations from in-situ drill-hole samples. Radiation Measurements 81, 98-103.

Singarayer, J.S., Bailey, R., 2003. Further investigations of the quartz optically stimulated luminescence components using linear modulation. Radiation Measurements 37, 451458.

Spooner, N.A., Questiaux, D.G., 2000. Kinetics of red, blue and UV thermoluminescence and optically-stimulated luminescence from quartz. Radiation Measurements 32, 659-666.

740 Spooner, N.A., Franklin, A.D., 2002. Effect of the heating rate on the red TL of quartz. Radiation Measurements 35, 59-66.

Stoneham, D., Stokes, S., 1991. An investigation of the relationship between the $110{ }^{\circ} \mathrm{C}$ TL peak and optically stimulated luminescence in sedimentary quartz. Nuclear Tracks and Radiation Measurements 18, 119-123.

Sunta, C.M., David M., 1982. Firing temperature of pottery from pre-dose sensitization of TL. PACT 6, 460-467.

750 Vaccaro, G., Panzeri, L., Paleari, S., Martini, M., Fasoli, M., 2017. EPR investigation of the role of germanium centers in the production of the $110^{\circ} \mathrm{C}$ thermoluminescence peak in quartz

Quaternary Geochronology 39, 99-104. 
755 Visocekas, R., 1985. Tunnelling radiative recombination in labradorite: Its association with anomalous fading of thermoluminescence. Nuclear Tracks and Radiation Measurements $10,521-529$.

Watson, I., Aitken, M., 1985. Firing temperature analysis using the $110^{\circ} \mathrm{C}$ TL peak of quartz Nuclear Tracks and Radiation Measurements 10, 517-520.

Wickham, H., 2009. ggplot2: Elegant Graphics for Data Analysis. Springer, New York. http://ggplot2.org

765 Wintle, A.G., 1973. Anomalous fading of thermoluminescence in mineral samples. Nature $245,143-144$.

Wintle, A.G., 1975. Thermal quenching of thermoluminescence in quartz. Geophysical Journal. Royal Astronomical Society 41, 107-113.

770

Zimmerman, J., 1971. The radiation-induced increase of the $100{ }^{\circ} \mathrm{C}$ thermoluminescence sensitivity of fired quartz. Journal of Physics C: Solid State Physics 4, 3265-3276. 
Table 1: Published values for trapping parameters $E$ and $s$ for the $110^{\circ} \mathrm{C}$ TL peak of quartz.

Table 2 Technical parameters relevant for the interlaboratory comparison measurements (FI: sample carriers manufactured by Freiberg Instruments).

Table 3 Summary of $E$ and $s$ values obtained with the isothermal decay and the Hoogenstraaten method as well as calculated lifetimes $\tau$ at $20^{\circ} \mathrm{C}$.

Table 4 Summary of average values of $E, s$ and $\tau$ across all laboratories derived from three applied methods. Given $E$ values are calculated as the arithmetic average from individual values, the cited uncertainty range ( $1 \sigma$ confidence interval) corresponds to the standard deviation (hence reflecting the variety of individual values across laboratories). The average $s$ value is derived from the geometric mean; due to the wide spread of values (assumed lognormal distribution for isothermal decay and Hoogenstraaten method) no uncertainty range is presented. The average lifetime $\tau$ is calculated from the average $E$ and $s$ values, while $\tau_{\mathrm{s}}$ represents the (unweighted) average of individual lifetime values from each laboratory (given with standard deviation). The low $\tau$ values for the initial rise method (compared to $\tau_{\mathrm{s}}$ ) can be explained by the non-lognormal distribution of individual $s$ values, which may also affect their average value. $n$ denotes the number of contributing individual pairs of $E$ and $s$.

Fig. 1 Normalised TL glow curves up to $160^{\circ} \mathrm{C}$ for the FB reference quartz (average curves of five aliquots each), as measured in eight laboratories using two different heating rates $q=$ $0.1 \mathrm{~K} \mathrm{~s}^{-1}$ (a) and $q=5 \mathrm{~K} \mathrm{~s}^{-1}$ (b).

Fig. 2 Examples of TL glow curves of five aliquots recorded in one laboratory (reader 374) at a heating rate of $5 \mathrm{~K} \mathrm{~s}^{-1}$. The dashed lines indicate the maximum difference in TL peak temperatures of $\sim 18 \mathrm{~K}$.

805 Fig. 3 (a) Example plot of isothermal TL recorded at different temperatures (reader 150), (b) exemplary plot of the logarithmic inverse of the lifetime $\tau$ (inferred from isothermal decay curves) against $1 / k_{\mathrm{B}} T$, from which $E$ and $s$ can be determined (reader 154). Five aliquots were repeatedly measured at different isothermal holding temperatures. 
810 Fig. 4 Graphical summary of $E, s$ and $\tau$ values (subplots a, b and c, respectively) derived from the isothermal decay experiments. Error bars of $E$ and $s$ values represent $1 \sigma$ uncertainties resulting from a fit to data from five aliquots per laboratory in an Arrhenius plot. The $1 \sigma$ uncertainty of $\tau$ as shown in (c) was obtained by propagating the uncertainties of $E$ and $s$ accordingly.

Fig. 5 Summary of results of the IR method. Thermal activation energy $E$ (subplot a), frequency factor $s$ (subplot b) and lifetime $\tau$ (at $20{ }^{\circ} \mathrm{C}$; subplot c) are shown as boxplots for each laboratory and heating rate employed. Each boxplot represents five measured aliquots.

820 Fig. 6 Example of a Hoogenstraaten plot for five aliquots of the FB reference quartz (reader $351)$.

Fig. 7 Graphical summary of $E, s$ and $\tau$ values (subplots a, b and c, respectively) derived from the Hoogenstraaten method. Error bars of $E$ and $s$ values represent $1 \sigma$ uncertainties resulting 825 from a fit to data from five aliquots per laboratory in a Hoogenstraaten plot. The $1 \sigma$ uncertainty of $\tau$ as shown in (c) was obtained by propagating the uncertainties of $E$ and $s$ accordingly.

Fig. 8 Direct determination of the lifetime by recording phosphorescence following artificial irradiation of $1 \mathrm{~Gy}$ (readers 150 and 11-std-01-0001). 
Table 1: Published values for trapping parameters $E$ and $s$ for the $110^{\circ} \mathrm{C}$ TL peak of quartz.

\begin{tabular}{|c|c|c|c|c|c|c|c|c|}
\hline $\begin{array}{l}E \\
{[\mathrm{eV}]}\end{array}$ & $\begin{array}{l}s \\
{[1 / \mathrm{s}]}\end{array}$ & $\begin{array}{l}\tau\left(20^{\circ} \mathrm{C}\right) \\
{[\mathrm{min}]}\end{array}$ & Material & Detection filter & Method $^{\text {a }}$ & $\begin{array}{l}\text { Heating rate } \\
{[\mathrm{K} / \mathrm{s}]}\end{array}$ & Equipment & Reference \\
\hline $0.99 \pm 0.02$ & - & - & Quartz from Romano-British pottery & Corning $7-51+$ HA3 & IR, ID, VHR & Unknown & Custom & Wintle (1975) \\
\hline 0.99 & $8.30 \mathrm{E}+12$ & 212 & Unknown & Unknown & Unknown & Unknown & Custom & Fleming (1979) \\
\hline 0.8 & $2.70 \mathrm{E}+09$ & 352 & Unknown & Unknown & IR & Unknown & Unknown & Aitken (1985) \\
\hline $1.05 \pm 0.03$ & $4.51 \mathrm{E}+12$ & 4195 & Sawyer Premium Q synthetic quartz unannealed & HA3 & IR & 1 & Custom & Petrov and Bailiff (1995) \\
\hline $0.99 \pm 0.03$ & $4.65 \mathrm{E}+13$ & 38 & Sawyer Premium Q synthetic quartz annealed & HA3 & IR & 1 & Custom & Petrov and Bailiff (1995) \\
\hline 0.89 & $3.80 \mathrm{E}+11$ & 88 & Quartz from Australia, Morocco and UK & Hoya U340 & VHR & - & Risø DA-15, Alldred glow oven & Spooner and Questiaux (2000) \\
\hline 0.82 & $4.30 \mathrm{E}+10$ & 49 & Quartz from Australia, Morocco and UK & Hoya U340 & ID & Unknown & Risø DA-15, Alldred glow oven & Spooner and Questiaux (2000) \\
\hline $0.6-1.0$ & - & - & 1 synthetic, 12 different natural quartz samples & Unknown & GCD & 2 & Harshaw 2000A-B TLD & Pagonis et al. (2002) \\
\hline $0.73 \pm 0.06$ & - & - & Merck quartz, no pre-dose & Hoya U340 & GCD & 1 & Risø DA-20 & Kaya Keles et al. (2016) \\
\hline $0.60 \pm 0.03$ & - & - & Merck quartz, no pre-dose & BG39 + Corning 7-59 & GCD & 1 & Risø DA-20 & Kaya Keles et al. (2016) \\
\hline $0.65 \pm 0.05$ & - & - & Merck quartz, no pre-dose & BG39 & GCD & 1 & Risø DA-20 & Kaya Keles et al. (2016) \\
\hline $0.69 \pm 0.06$ & - & - & Merck quartz, no pre-dose & Corning 7-59 & GCD & 1 & Risø DA-20 & Kaya Keles et al. (2016) \\
\hline $0.75 \pm 0.06$ & - & - & Merck quartz, with pre-dose & Hoya U340 & GCD & 1 & Risø DA-20 & Kaya Keles et al. (2016) \\
\hline $0.63 \pm 0.04$ & - & - & Merck quartz, with pre-dose & BG39 + Corning 7-59 & GCD & 1 & Risø DA-20 & Kaya Keles et al. (2016) \\
\hline $0.68 \pm 0.06$ & - & - & Merck quartz, with pre-dose & BG39 & GCD & 1 & Risø DA-20 & Kaya Keles et al. (2016) \\
\hline $0.72 \pm 0.06$ & - & - & Merck quartz, with pre-dose & Corning 7-59 & GCD & 1 & Risø DA-20 & Kaya Keles et al. (2016) \\
\hline $0.79 \pm 0.05$ & $4.00 \pm 1.00 \mathrm{E}+10$ & 16 & Natural, hydrothermal, hyaline single crystal & Hoya U340 & GCD & 5 & Risø DA-20 & Vaccaro et al. (2017) \\
\hline $0.76 \pm 0.07$ & $2.30 \pm 0.80 \mathrm{E}+09$ & 85 & Natural, hydrothermal, hyaline single crystal & Hoya U340 & ID & 5 & Risø DA-20 & Vaccaro et al. (2017) \\
\hline $0.80 \pm 0.14^{b}$ & & & & & & & & \\
\hline
\end{tabular}

a $\mathrm{IR}=$ initial rise, $\mathrm{ID}=$ isothermal decay, $\mathrm{VHR}=$ varying heating rates, $\mathrm{GCD}=$ glow curve deconvolution

${ }^{\mathrm{b}}$ Arithmetic average value and $1 \sigma$ standard deviation (excluding the $E$ values from Pagonis et al. 2002). 
Table 2 Technical parameters relevant for the interlaboratory comparison measurements (FI: sample carriers manufactured by Freiberg Instruments).

\begin{tabular}{|c|c|c|c|c|c|c|}
\hline Reader ID & Reader & Sample carrier & $\begin{array}{l}\text { Thickness } \\
{[\mathrm{mm}]}\end{array}$ & $\begin{array}{l}\text { Dose rate } \\
{[\mathrm{Gy} / \mathrm{s}]}\end{array}$ & Detection filters & PMT \\
\hline 267 & Risø TL/OSL DA-20 & Stainless steel discs & 0.3 & 0.11 & Hoya U340 & EMI 9235QB \\
\hline 150 & Risø TL/OSL DA-20 & Aluminium cups & 0.1 & 0.12 & Hoya U340 & EMI 9235QB \\
\hline 11 -std-01-0001 & Freiberg Instruments lexsyg research & Stainless steel cups (FI) & 0.3 & 0.055 & Schott BG3 + Delta BP365/50 EX & Hamamatsu H7360-02 \\
\hline 351 & Risø TL/OSL DA-20 & Stainless steel discs & 0.3 & 0.058 & Hoya U340 & EMI 9235QB \\
\hline 12-re-01-0007 & Freiberg Instruments lexsyg research ${ }^{\mathrm{a}}$ & Stainless steel cups (FI) & 0.49 & 0.056 & Hoya U340 + Delta BP365/50 EX & Hamamatsu H7360-02 \\
\hline 14-16-01-0008 & Freiberg Instruments lexsyg smart ${ }^{\mathrm{a}}$ & Stainless steel cups (FI) & 0.49 & 0.185 & Hoya U340 + Delta BP365/50 EX & Hamamatsu H7360-02 \\
\hline 6220 & Risø TL/OSL DA-15 & Stainless steel discs & 0.25 & 0.12 & Hoya U340 & EMI 9235QB \\
\hline 374 & Risø TL/OSL DA-20 & Stainless steel discs & 0.5 & 0.12 & Hoya U340 & EMI 9235QB \\
\hline 154 & Risø TL/OSL DA-20 & Aluminium cups & 0.1 & 0.12 & Hoya U340 & EMI 9235QB \\
\hline 154 (Risø discs) & Risø TL/OSL DA-20 & Stainless steel discs & 0.3 & 0.12 & Hoya U340 & EMI 9235QB \\
\hline 320 & Risø TL/OSL DA-20 & Stainless steel discs & 0.3 & 0.12 & Hoya U340 & EMI 9235QB \\
\hline
\end{tabular}

${ }^{a}$ Isothermal TL measurements were carried out with a lexsyg research device, while other measurements (IR, Hoogenstraaten) were done with a lexsyg smart reader. 
Table 3 Summary of $E$ and $s$ values obtained with the isothermal decay and the Hoogenstraaten method as well as calculated lifetimes $\tau$ at $20{ }^{\circ} \mathrm{C}$.

Isothermal decay method

\begin{tabular}{llll}
\hline Reader ID & $\boldsymbol{E}[\mathbf{e V}]$ & $\boldsymbol{s}[\mathbf{1} / \mathbf{s}]$ & $\boldsymbol{\tau}[\mathbf{m i n}]$ \\
\hline 267 & $0.86 \pm 0.01$ & $1.9 \pm 0.7 \mathrm{e}+11$ & $60 \pm 30$ \\
150 & $0.734 \pm 0.008$ & $1.2 \pm 0.3 \mathrm{e}+09$ & $60 \pm 30$ \\
11 -std-01-0001 & $0.989 \pm 0.007$ & $2.9 \pm 0.7 \mathrm{e}+13$ & $60 \pm 20$ \\
351 & $0.957 \pm 0.004$ & $7.91 \pm 1.13 \mathrm{e}+12$ & $60 \pm 10$ \\
$12-$ re-01-0007 & $1.015 \pm 0.007$ & $7.3 \pm 1.7 \mathrm{e}+13$ & $70 \pm 20$ \\
6220 & $0.901 \pm 0.007$ & $8 \pm 2 \mathrm{e}+11$ & $70 \pm 30$ \\
374 & $0.856 \pm 0.004$ & $1.7 \pm 0.2 \mathrm{e}+11$ & $50 \pm 10$ \\
154 & $0.862 \pm 0.009$ & $3.2 \pm 1.1 \mathrm{e}+11$ & $40 \pm 20$ \\
320 & $0.907 \pm 0.005$ & $1.1 \pm 0.2 \mathrm{e}+12$ & $60 \pm 20$ \\
\hline
\end{tabular}

Hoogenstraaten method

\begin{tabular}{llll}
\hline Reader ID & $\boldsymbol{E}[\mathbf{e V}]$ & $\boldsymbol{s}[\mathbf{1 / s}]$ & $\boldsymbol{\tau}$ [min] \\
\hline 267 & $0.52 \pm 0.02$ & $5 \pm 3 \mathrm{e}+05$ & $30 \pm 30$ \\
150 & $0.70 \pm 0.01$ & $3.6 \pm 1.2 \mathrm{e}+08$ & $50 \pm 20$ \\
11 -std-01-0001 & $0.75 \pm 0.02$ & $7 \pm 4 \mathrm{e}+09$ & $20 \pm 20$ \\
351 & $0.892 \pm 0.008$ & $8 \pm 2 \mathrm{e}+11$ & $50 \pm 20$ \\
$14-16-01-0008$ & $0.64 \pm 0.02$ & $5 \pm 4 \mathrm{e}+07$ & $30 \pm 30$ \\
6220 & $0.67 \pm 0.02$ & $2 \pm 1 \mathrm{e}+08$ & $30 \pm 30$ \\
374 & $0.64 \pm 0.02$ & $4 \pm 3 \mathrm{e}+07$ & $30 \pm 30$ \\
154 & $1.077 \pm 0.007$ & $10 \pm 3 \mathrm{e}+14$ & $60 \pm 20$ \\
320 & $0.79 \pm 0.02$ & $1.5 \pm 0.8 \mathrm{e}+10$ & $40 \pm 30$ \\
267 & $0.768 \pm 0.013$ & $6 \pm 3 \mathrm{e}+09$ & $40 \pm 30$ \\
\hline
\end{tabular}


Table 4 Summary of average values of $E, s$ and $\tau$ across all laboratories derived from three applied methods. Given $E$ values are calculated as the arithmetic average from individual values, the cited uncertainty range ( $1 \sigma$ confidence interval) corresponds to the standard deviation (hence reflecting the variety of individual values across luminescence readers). The average $s$ value is derived from the geometric mean; due to the wide spread of values (assumed lognormal distribution for isothermal decay and Hoogenstraaten method) no uncertainty range is presented. The average lifetime $\tau$ is calculated from the average $E$ and $s$ values, while $\tau_{\mathrm{s}}$ represents the (unweighted) average of individual lifetime values from each laboratory (given with standard deviation). The low $\tau$ values for the initial rise method (compared to $\tau_{\mathrm{s}}$ ) can be explained by the non-lognormal distribution of individual $s$ values, which may also affect their average value. $n$ denotes the number of contributing individual pairs of $E$ and $s$.

\begin{tabular}{lllllll}
\hline & Isothermal decay & \multicolumn{3}{c}{ Initial rise $(n=10)$} & Hoogenstraaten \\
\cline { 3 - 6 } & $(n=9)$ & $0.5 \mathrm{~K} / \mathrm{s}$ & $1 \mathrm{~K} / \mathrm{s}$ & $2 \mathrm{~K} / \mathrm{s}$ & $3 \mathrm{~K} / \mathrm{s}$ & $0.83 \pm 0.14$ \\
\hline$E[\mathrm{eV}]$ & $0.90 \pm 0.08$ & $0.9 \pm 0.2$ & $0.9 \pm 0.1$ & $0.87 \pm 0.12$ & $0.9 \pm 0.1$ & $8.1 \mathrm{e}+10$ \\
$s\left[\mathrm{~s}^{-1}\right]$ & $8.3 \mathrm{e}+11$ & $1.3 \mathrm{e}+12$ & $6.1 \mathrm{e}+11$ & $4.0 \mathrm{e}+11$ & $4.8 \mathrm{e}+11$ & 46 \\
$\tau[\mathrm{min}]$ & 56 & 38 & 37 & 38 & 69 & $410 \pm 60$ \\
$\tau_{\mathrm{s}}[\mathrm{min}]$ & $57 \pm 9$ & $60 \pm 30$ & $70 \pm 30$ & $80 \pm 40$ & $110 \pm 14$ \\
\hline
\end{tabular}


25000 -

Reader 374

Aliquot

20000 - - AQ 1

- AQ 2

AQ 3

$\bar{U} 15000$ - $\quad A Q 4$

- AQ 5

5000 -

0 -

50

100

150

\section{Temperature $\left[{ }^{\circ} \mathrm{C}\right]$}




\section{平}

$\sum_{\frac{0}{\omega}}^{0.9-} \Phi$

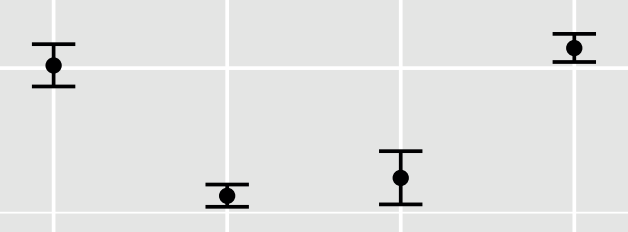

$0.8-$

\section{포}

v 150

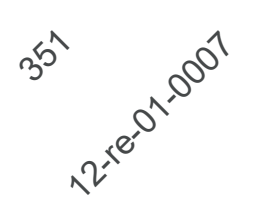

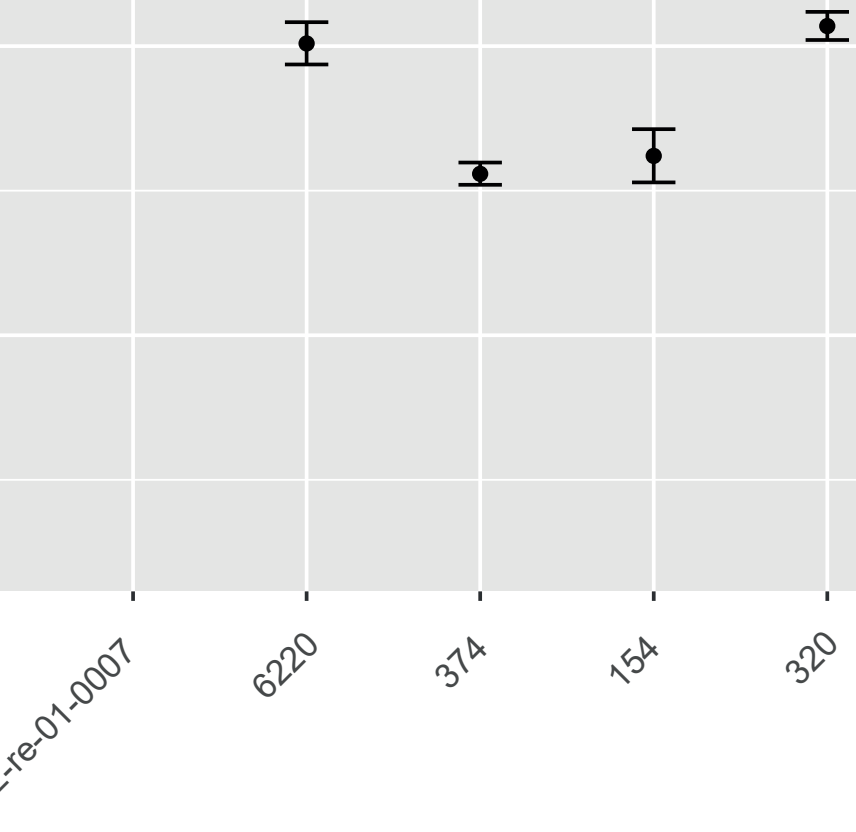

b

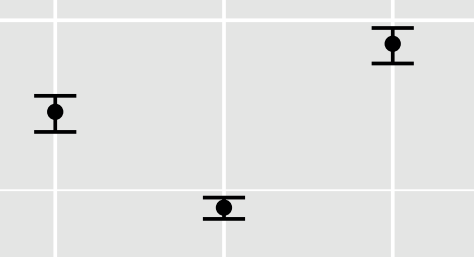

$\frac{\bar{s}}{\stackrel{s}{s}}$

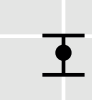

포

$\mp \Phi$

$1 e+10-$

$\Phi$

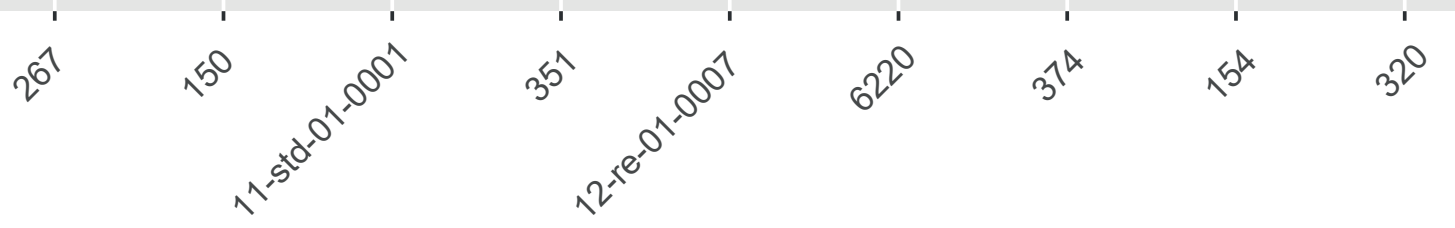

C

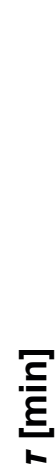

$\Phi$

ㅎ
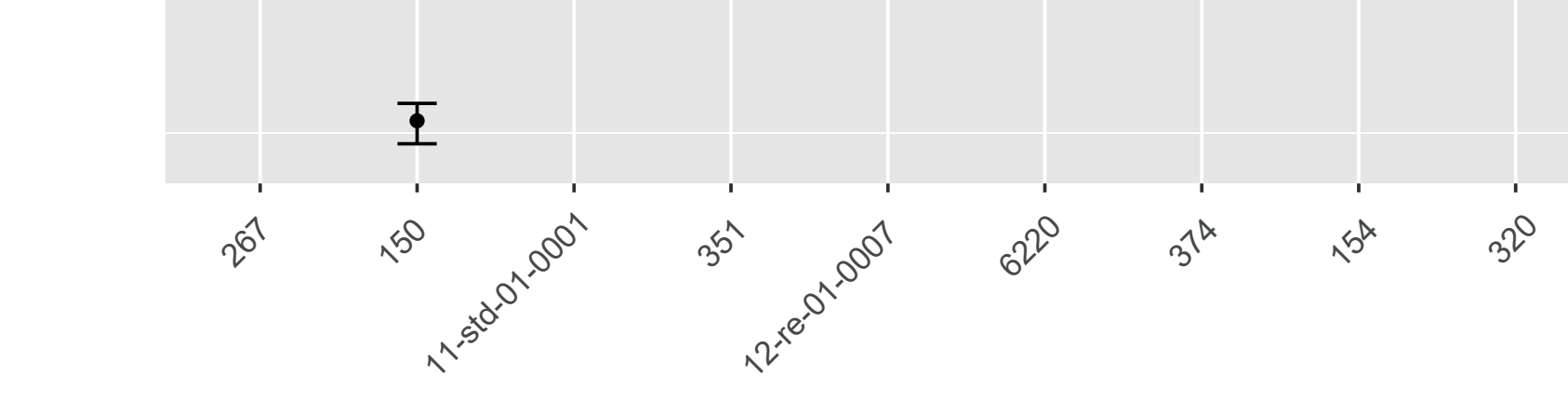

$2^{6}$ र

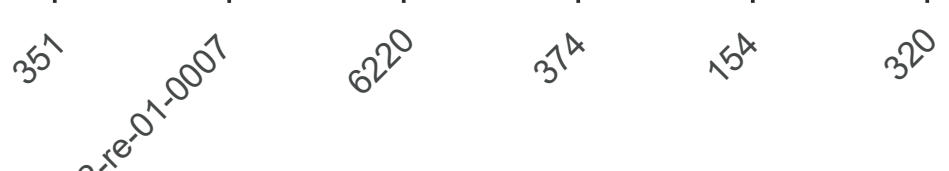


$3-$

만

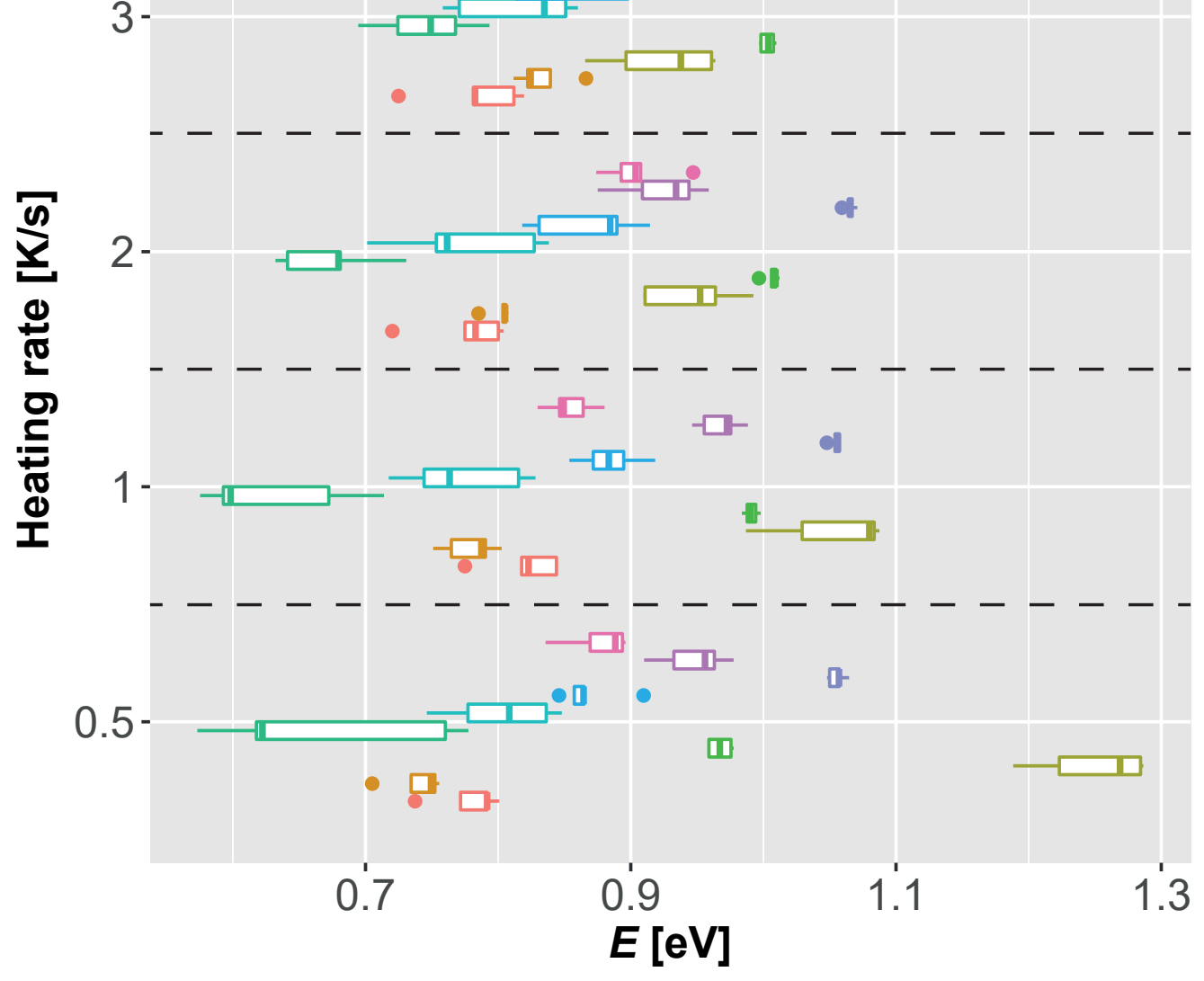

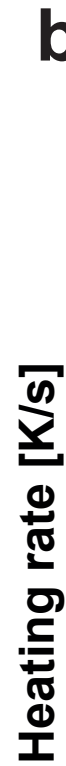

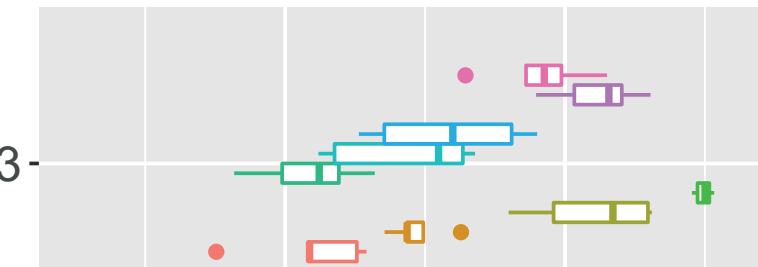

|

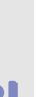
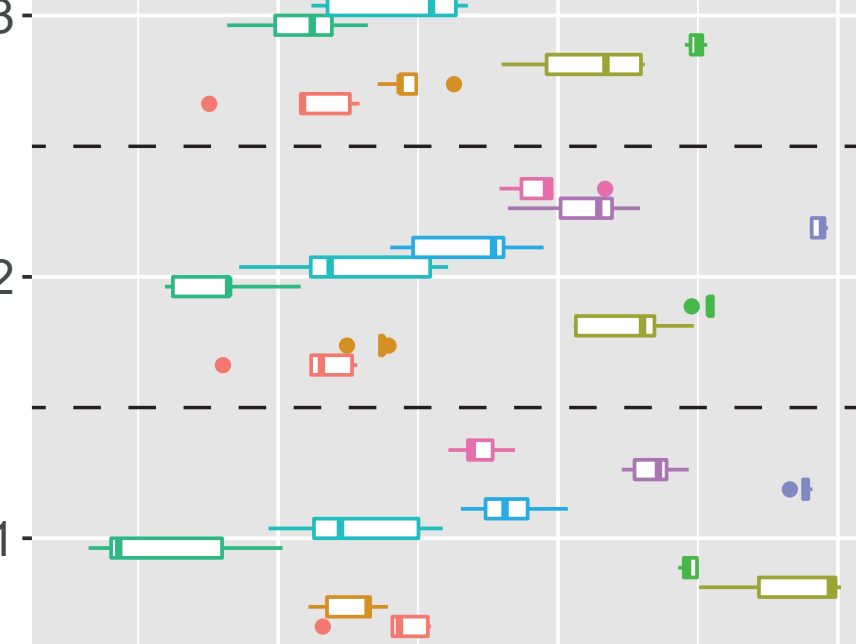

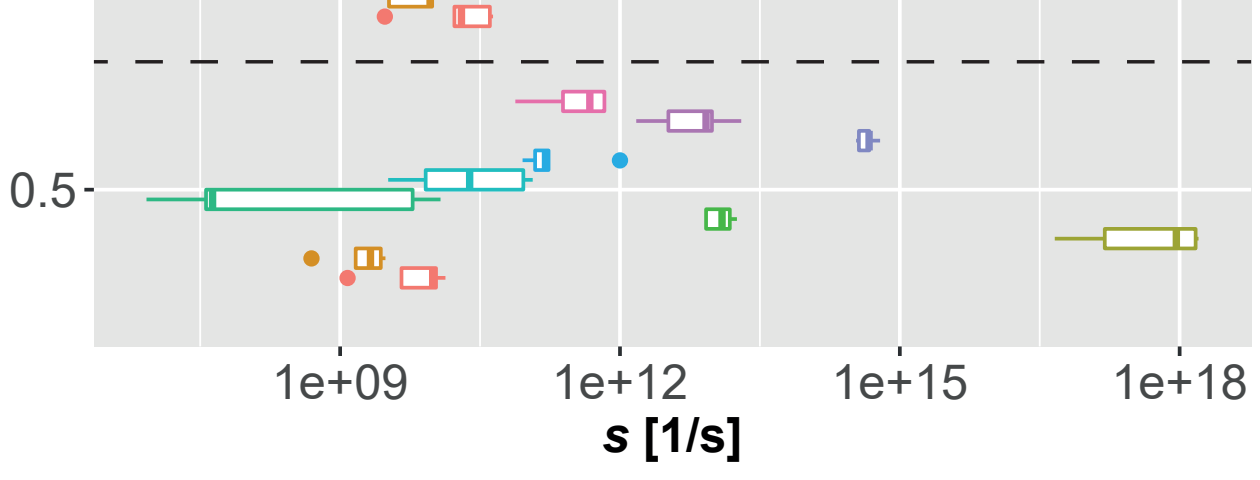

C

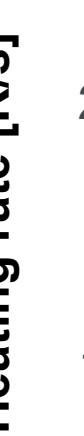

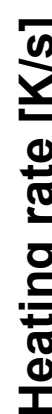
3 
Reader 351

14 -

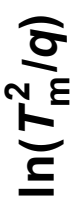

12

10 -

Aliquot

- 1

- 2

- 3

- 4

5

32

34

36

$1 / k_{\mathrm{B}} T_{\mathrm{m}}$ 


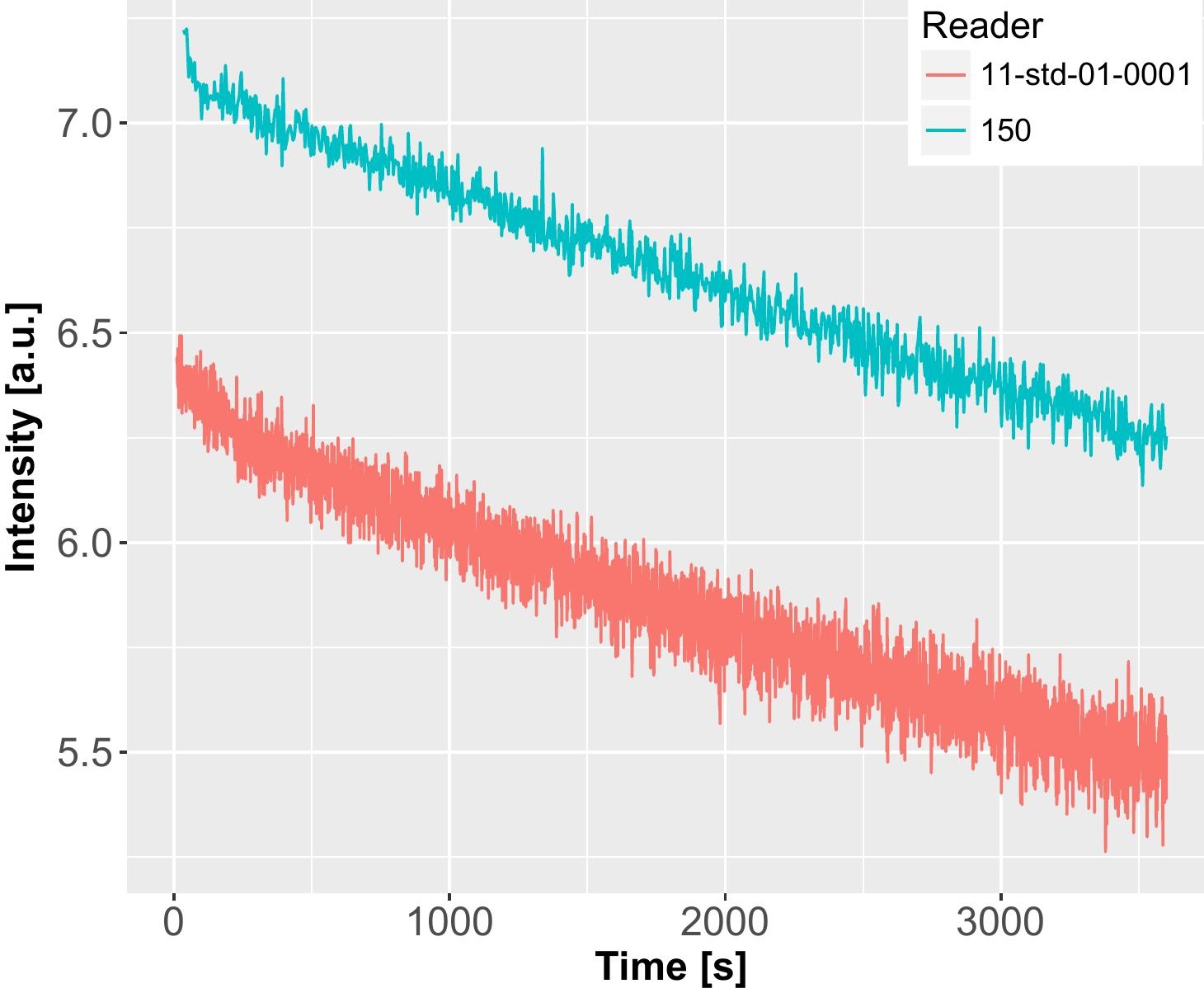

